\title{
Energy Management Optimization for Cellular Networks under Renewable Energy Generation Uncertainty
}

\author{
Nadhir Ben Rached, Student Member, IEEE, Hakim Ghazzai, Member, IEEE, Abdullah Kadri, Senior \\ Member, IEEE, and Mohamed-Slim Alouini, Fellow, IEEE
}

\begin{abstract}
The integration of renewable energy (RE) as an alternative power source for cellular networks has been deeply investigated in literature. However, RE generation is often assumed to be deterministic; an impractical assumption for realistic scenarios. In this paper, an efficient energy procurement strategy for cellular networks powered simultaneously by the smart grid (SG) and locally deployed RE sources characterized by uncertain processes is proposed. For a one-day operation cycle, the mobile operator aims to reduce its total energy cost by optimizing the amounts of energy to be procured from the local RE sources and SG at each time period. Additionally, it aims to determine the amount of extra generated RE to be sold back to SG. A chance constrained optimization is first proposed to deal with the RE generation uncertainty. Then, two convex approximation approaches: Chernoff and Chebyshev methods, characterized by different levels of knowledge about the RE generation, are developed to determine the energy procurement strategy for different risk levels. In addition, their performances are analyzed for various daily scenarios through selected simulation results. It is shown that the higher complex Chernoff method outperforms the Chebyshev one for different risk levels set by the operator.
\end{abstract}

Index Terms-Cellular networks, chance constrained optimization, convex approximation, renewable energy generation uncertainty.

\section{INTRODUCTION}

Over the last decade, mobile traffic has grown rapidly due to the emergence of various mobile devices applications that enjoyed widespread popularity and usage in addition to the rise of the internet-of-things (IoT) concept [1]. From 2010 to 2014, global mobile data traffic has increased by 12 times and is also expected to reach, by 2020, 10 times as much as the global mobile traffic in 2014 [2], [3]. However, this growth is envisioned to demand much higher energy consumption than today's level and hence it negatively affects the environment due to the increase of carbon dioxide $\left(\mathrm{CO}_{2}\right)$ emission [4]. In fact, $\mathrm{CO}_{2}$ emissions are also expected to increase by a growth of $6 \%$ every year through 2020 [5]. Therefore, a pressing

A part of this work has been accepted for publication in IEEE Global Communications Conference (Globecom 2016).

This work was made possible by grant NPRP \# 6-001-2-001 from the Qatar National Research Fund (a member of The Qatar Foundation). The statements made herein are solely the responsibility of the authors.

Nadhir Ben Rached and Mohamed-Slim Alouini are with King Abdullah University of Science and Technology (KAUST), Thuwal, Makkah Province, Saudi Arabia. E-mails: \{nadhir.benrached, slim.alouini\}@kaust.edu.sa.

Hakim Ghazzai and Abdullah Kadri are with Qatar Mobility Innovations Center (QMIC), Qatar University, Doha, Qatar. E-mails: \{hakimg, abdullahk\}@qmic.com. need to reduce $\mathrm{CO}_{2}$ emissions of wireless cellular networks is imposed [6].

The next generation power grid, known as smart grid (SG), enables the use of developed information technologies through which it efficiently delivers power to its customers [7]. The power management within SG is performed such that various economical and environmental goals are met via the reduction of greenhouse gas emissions and the optimal adjustment of the supplied power depending on the customers' needs [8]. It offers the possibility to deliver electricity more cost-effectively with enabling the active involvement of the customers in the procurement decision [9]. Furthermore, it enables the consumer to sell the extra energy generated locally to the market and allows the massive integration of intermittent renewable energy (RE) sources such as wind, solar, and hydro [10]-[12]. This is in accordance with the objectives of the International Energy Agency aiming to accelerate the exploitation of these alternative sources to reach $31 \%$ of the total power generation by 2035 [13].

Integrating RE sources as alternative way to power cellular networks has been deeply investigated in the literature [14][16]. The introduction of RE generators such as solar panels, wind turbines, etc, at the base station (BS) site offers to mobile operators the possibility to obtain green and low cost electricity. It also reduces its dependence on external energy retailers and help downward pressure on energy prices. The management of energy procurement from SG has also been widely discussed in literature. In this scenario, RE sources are either assumed to be owned by the mobile operator itself or by an external entity [17]-[19]. An optimization framework combining the BS on/off switching strategy with the energy procurement management has been proposed in [17]. In [18], a multi-stage energy allocation problem and a multi-BSs energy balancing problem are developed to achieve on-grid energy savings. In [19], the energy procurement from SG is optimized based on average statistics of the network. A stochastic geometry based approach is developed to determine the average energy requirement of the networks and optimize the demand side management (DSM) accordingly. The obtained results had shown significant $\mathrm{CO}_{2}$ reductions and cost savings can be achieved thanks to the introduction of RE and the optimization of the DSM of cellular networks from SG. However, most of these studies have assumed deterministic RE generation models which are not applicable in practical scenarios mainly the ones requiring pre-planned $\mathrm{RE}$ management or aiming at 
estimating energy cost at the future. Indeed, power generation from renewable sources is generally random in time and space due to various environmental factors [20]. For instance, photovoltaic energy depends on the amount of solar irradiance, the size of the solar panel, the shadow level, and energy conversion efficiency, etc. Hence, the BSs powered by RE sources may sometimes have an excess of energy while at other times, they may be energy deficient. Hence, handling this uncertainty in the energy management framework for cellular networks presents an important challenge that has received interests in literature [14], [21]-[24]. The authors of [23], [24], for instance, have employed stochastic programming for adaptive power management under various uncertainties including renewable power generation. However, their proposed approach is only suitable when the distribution of the uncertainty parameter is discrete. Moreover, even for discrete distributions, the complexity of their approach increases as the number of possible outcomes increases. Some other studies, such as [21] and [22], have investigated the energy management from another perspective with the objective to optimize the resource allocation while taking into account hybrid energy sources including RE ones. Transmit power and carrier allocation schemes are developed in order to optimize communication utility metrics such as throughput maximization while considering uncertainty of the RE generation. In this paper, however, we are dealing with the energy procurement of cellular networks powered by uncertain RE sources and conventional electricity with the objective to minimize the cost of energy procurement [25].

The objective of this study is to determine the amount of energy to be procured by each BS belonging to the cellular network from each energy source, namely; the fossil fuel source existing in SG and the RE generated locally. Moreover, in case of excess of RE generation, we aim to determine the extra amount of RE to be sold to the electrical grid in order to compensate the energy cost. A time-varied scenario is taken into consideration in the proposed energy procurement framework in the sense that the procurement at a given time period depends on the variation of the SG price, the RE generation during the whole operation cycle, and the storage capacity at the BS level. The objective is to make accurate procurement decision in order to minimize the cost of total energy. For instance, if we assume that during any time slot the operator knows the RE generation in future time slots will be low, then the optimization framework will be inclined towards conserving the use of energy from the battery in the current time slot in order to save it for the future and vice versa. Hence, the proposed approach offers the possibility for mobile operators to pre-plan the use of the available RE over future time slots. This allows them to predict the cost of the network energy consumption according to the traffic profile of their networks and to adjust their energy procurement according to the RE availability and the variation of SG prices. In this paper, we propose to deal with the randomness of the RE generation by formulating chance constrained optimization problems. However, this probabilistic approach could yield either to non-convex constraints or introduce some difficulties regarding their evaluations. To avoid such problems, we employ conservative convex approximation approaches. Two methods are applied: the Chernoff- and the Chebyshev-based approaches. The former assumes the knowledge of the moment generating functions (MGFs) of the RE random variables (RVs) whereas the latter is only based on the knowledge of the first and second moments of the RE distribution. Moreover, for a given confidence level, we investigate which of these methods is efficient in terms of energy procurement decision. More precisely, while both approaches are constructed such that a given confidence level is satisfied, we identify the more conservative method that procures the energy less efficiently than the other one. Selected simulation results analyzing the effect of the uncertainty and comparing the Chernoff- and the Chebyshev-based approaches are provided for various scenarios.

The paper is organized as follows. The system model is described in Section II. In Section III, the problem formulation based on the chance constrained optimization approach is presented. In Section IV, convex approximations of the formulated problem are investigated using Chernoff and Chebyshev approximations. Selected simulation results using these two approaches are presented in Section V for different scenarios. Finally, conclusions are drawn in Section VI.

\section{SySTEM MODEL}

We consider a cellular network consisting of $N$ BSs as illustrated in Fig. 1. We aim to optimize the energy procurement at each BS level for one-day operation cycle divided into $T$ time intervals of duration $\Delta t$. We denote the energy procured by the mobile operator to power its BS $n \in\{1, \ldots, N\}$ from an external retailer during time period $i \in\{1, \ldots, T\}$ by $q_{i, n}^{1}$. The corresponding unit price at the $i^{\text {th }}$ time period is denoted by $c_{i}^{1}$. This price can vary during the day depending on a certain strategy followed by the energy retailer. Moreover, each BS has its own internal retailer, i.e., RE generator, that produces an amount of energy, denoted by $Q_{i, n}^{0}$, which is assumed to be free of charge. We assume that each internal retailer has a maximum storage capacity $\bar{C}_{n}$. The amount of energy procured by a BS $n$ at the $i^{t h}$ time period from its own internal retailer is denoted by $q_{i, n}^{0}$. As the cellular network is interconnected to the SG, we consider that each BS $n$ has the ability to sell an amount of energy $\tilde{q}_{i, n}^{0}$ to the SG at each time period $i$ at a given price $c_{i}^{0}$. Without loss of generality, we assume that $c_{i}^{0}$ is strictly less than $c_{i}^{1}$ for all $i \in\{1, \ldots, T\}$.

The average energy consumption at time period $i$ of $\mathrm{BS} n$ is denoted by $E_{i, n}$ and can be expressed as follows [26]:

$$
E_{i, n}=\left(a P_{u} \bar{N}_{n, i}+b\right) \Delta t,
$$

where $a$ is a factor that scales with the radiated power due to amplifier and feeder losses, $b$ models an offset of site power which is consumed independently of the average transmit power, $P_{u}$ is the consumed power per unit of traffic that we assume to be fixed and uniform, and $\bar{N}_{n, i}$ is the average traffic profile corresponding to the $n^{t h} \mathrm{BS}$ and during the $i^{\text {th }}$ time period and is given by:

$$
\bar{N}_{n, i}=\frac{1}{\Delta t} \int_{(i-1) \Delta t}^{i \Delta t} N_{n}(t) d t
$$




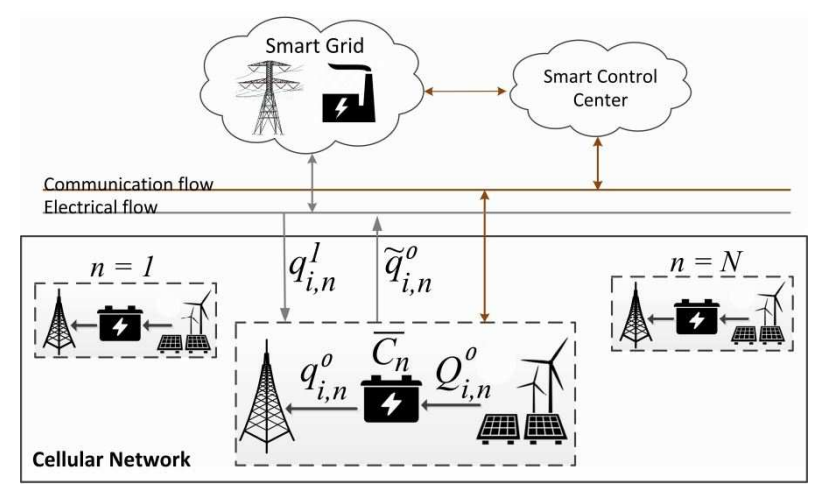

Fig. 1: Energy management for cellular networks powered by SG and locally deployed RE sources.

where $N_{n}(t)$ is the traffic profile of the $n^{\text {th }}$ BS.

The energy procurement decision is centrally managed by a smart control center (SCC) collecting all needed information from the network's BSs and the SG as shown in Fig. 1. More precisely, after receiving data from the different actors, such as energy price strategy of the smart grid, RE information, BS energy consumption, etc., the SCC determines the amount of energy to be procured by each BS during the whole operation cycle.

\section{Problem Formulation}

In this section, we formulate the optimization problem aiming to maximize the mobile operator's profit $P$ given as follows:

$$
P=\sum_{i=1}^{T} \sum_{n=1}^{N}\left(c_{i}^{0} \tilde{q}_{i, n}^{0}-c_{i}^{1} q_{i, n}^{1}\right) .
$$

This profit represents the summation of the individual profit of each BS during the one-day operation cycle. Note that $P$ corresponds to the revenue due to the energy exchange with the SG and can be negative depending on the amount of energy to be sold or bought. This maximization problem should be carried out under three constraints. i) The mobile operator has to fulfill the energy consumption constraint at each time period for all BSs. ii) For each BS $n$ and time period $i$, the total summation up to the time period $i$ of the amounts of energy consumed from the internal retailer, $q_{j, n}^{0}$, and the energy sold to the SG, $\tilde{q}_{j, n}^{0}$, where $j \in\{1, \ldots, i\}$, cannot exceed the generated energy of the internal retailer up to the $i^{t h}$ time period. This means that at each time period $i$, the used RE cannot exceed the amount of energy generated up to that time minus the amount of energy consumed in the previous time periods. (iii) Finally, for each internal retailer, the amount of the stored energy should not exceed the capacity $\bar{C}_{n}$. In a nutshell, the maximization problem can be expressed as follows:

$$
\begin{array}{ll}
\underset{\tilde{q}_{i, n}^{0}, q_{i, n}^{0}, q_{i, n}^{1}}{\operatorname{maximize}} & \sum_{i=1}^{T} \sum_{n=1}^{N}\left(c_{i}^{0} \tilde{q}_{i, n}^{0}-c_{i}^{1} q_{i, n}^{1}\right) \\
\text { subject to } & q_{i, n}^{0}+q_{i, n}^{1}=E_{i, n}, \forall i, n \\
& \sum_{j=1}^{i}\left(q_{j, n}^{0}+\tilde{q}_{j, n}^{0}\right) \leq \sum_{j=1}^{i} Q_{j, n}^{0}, \forall i, n, \\
& \sum_{j=1}^{i} Q_{j, n}^{0}-\sum_{j=1}^{i}\left(q_{j, n}^{0}+\tilde{q}_{j, n}^{0}\right) \leq \bar{C}_{n}, \forall i, n, \\
& q_{i, n}^{0}, \tilde{q}_{i, n}^{0}, q_{i, n}^{1} \geq 0, \forall i, n .
\end{array}
$$

Note that from constraint (7), we adopt a use and store strategy in the sense that the available energy from the internal retailer is used before being stored. It is also worth noting that the above optimization problem is separable such that it can be solved for each BS independently. This is because we assume that there is no dependence, among all BSs, in the procurement decision. This assumption is made because the traffic of each cell is independent from the other cells and the RE locally generated at each BS site is independent from what is generated at the other BS sites. Scenarios where dependence among BSs will be considered in the future extensions of this work. Thus, the mobile operator aims to solve for each BS $n$, where $n \in\{1, \ldots, N\}$, the following problem:

$$
\begin{array}{ll}
\underset{\tilde{q}_{i, n}^{0}, q_{i, n}^{0}, q_{i, n}^{1}}{\operatorname{maximize}} & \sum_{i=1}^{T}\left(c_{i}^{0} \tilde{q}_{i, n}^{0}-c_{i}^{1} q_{i, n}^{1}\right) \\
\text { subject to } & q_{i, n}^{0}+q_{i, n}^{1}=E_{i, n}, \forall i, \\
& \sum_{j=1}^{i}\left(q_{j, n}^{0}+\tilde{q}_{j, n}^{0}\right) \leq \sum_{j=1}^{i} Q_{j, n}^{0}, \forall i, \\
& \sum_{j=1}^{i} Q_{j, n}^{0}-\sum_{j=1}^{i}\left(q_{j, n}^{0}+\tilde{q}_{j, n}^{0}\right) \leq \bar{C}_{n}, \forall i, \\
& q_{i, n}^{0}, \tilde{q}_{i, n}^{0}, q_{i, n}^{1} \geq 0, \forall i .
\end{array}
$$

The resolution of the previous maximization problem could be easily performed through linear programming algorithms [27]. However, from a practical point of view, the RE generation is not deterministic due to, for instance, the randomness of wind and solar power generations. Thus, it is more practical to model the energy generated by each internal retailer by a random variable $(\mathrm{RV})$.

In the presence of uncertainty in $Q_{i, n}^{0}$, the second and third constraints of the maximization problem formulated earlier become random quantities and hence, the optimization problem has to be modified. Chance constrained optimization is a probabilistic approach that we can opt for in order to solve the maximization problem. By defining $\mathbf{q}=\left\{\tilde{q}_{i, n}^{0}, q_{i, n}^{0}, q_{i, n}^{1}\right\}_{i \in\{1, \ldots, T\}, n \in\{1, \ldots, N\}}$ and $\mathbf{Q}^{\mathbf{0}}=\left\{Q_{i, n}^{0}\right\}_{i \in\{1, \ldots, T\}, n \in\{1, \ldots, N\}}$ and denoting by $f_{i, n}\left(\mathbf{q}, \mathbf{Q}^{\mathbf{0}}\right)=\sum_{j=1}^{i} q_{j, n}^{0}+\tilde{q}_{j, n}^{0}-\sum_{j=1}^{i} Q_{j, n}^{0}$ and $g_{i, n}\left(\mathbf{q}, \mathbf{Q}^{\mathbf{0}}\right)=\sum_{j=1}^{i} Q_{j, n}^{0}-\sum_{j=1}^{i} q_{j, n}^{0}+\tilde{q}_{j, n}^{0}-\bar{C}_{n}$, the chance constrained optimization problem can be formulated 
as follows: $\forall n \in\{1, \ldots, N\}$

$\underset{\tilde{q}_{i, n}^{0}, q_{i, n}^{0}, q_{i, n}^{1}}{\operatorname{maximize}} \sum_{i=1}^{T}\left(c_{i}^{0} \tilde{q}_{i, n}^{0}-c_{i}^{1} q_{i, n}^{1}\right)$

subject to $(10),(13)$,

$$
P\left(\cap_{i}\left\{f_{i, n}\left(\mathbf{q}, \mathbf{Q}^{\mathbf{0}}\right) \leq 0, g_{i, n}\left(\mathbf{q}, \mathbf{Q}^{\mathbf{0}}\right) \leq 0\right\}\right) \geq \eta,
$$

where $0 \ll \eta<1$ is a confidence measure which is generally selected to be close to 1 in order to guarantee a safe network operation. To facilitate the resolution of the previous maximization problem, a more conservative approach can be considered instead. In fact, (15) can be re-written and upperbounded $\forall n \in\{1, \ldots, N\}$ as follows:

$$
\begin{aligned}
& P\left(\cup_{i}\left\{\left\{f_{i, n}\left(\mathbf{q}, \mathbf{Q}^{\mathbf{0}}\right)>0\right\} \cup\left\{g_{i, n}\left(\mathbf{q}, \mathbf{Q}^{\mathbf{0}}\right)>0\right\}\right\}\right) \\
& \leq \sum_{i} P\left(f_{i, n}\left(\mathbf{q}, \mathbf{Q}^{\mathbf{0}}\right)>0\right)+P\left(g_{i, n}\left(\mathbf{q}, \mathbf{Q}^{\mathbf{0}}\right)>0\right) .
\end{aligned}
$$

Let $\xi_{i, n}$ and $\nu_{i, n}$ be positive numbers satisfying $\sum_{i}\left(1-\xi_{i, n}\right)+\sum_{i}\left(1-\nu_{i, n}\right)=1-\eta, \forall n \in\{1, \ldots, N\}$, then, a conservative approximation of the optimization problem (14)-(15) can be given as follows: $\forall n \in\{1, \ldots, N\}$

$$
\begin{array}{ll}
\underset{\tilde{q}_{i, n}^{0}, q_{i, n}^{0}, q_{i, n}^{1}}{\operatorname{maximize}} & \sum_{i=1}^{T}\left(c_{i}^{0} q_{i, n}^{0}-c_{i}^{1} q_{i, n}^{1}\right) \\
\text { subject to } & (10),(13), \\
& P\left(f_{i, n}\left(\mathbf{q}, \mathbf{Q}^{\mathbf{0}}\right) \leq 0\right) \geq \xi_{i, n}, \forall i, \\
& P\left(g_{i, n}\left(\mathbf{q}, \mathbf{Q}^{\mathbf{0}}\right) \leq 0\right) \geq \nu_{i, n}, \forall i .
\end{array}
$$

It is worth mentioning that this probabilistic approach cannot be easily solved and requires the knowledge of a lot of information about the RE generation which are not always available in practice. For example, it necessitates the knowledge of the distributions of the sum of RVs that are out of reach in many practical scenarios. In this case, the use of Monte Carlo (MC) method to check the feasibility of a given point may be costly especially for values of $\xi_{i, n}$ or $\nu_{i, n}$ that are close to one. Moreover, it may happen that the feasible set, under these probabilistic constraints, is not convex. Hence, this leads to serious numerical problems and makes the optimal solution difficult to find. Therefore, we propose to focus on convex approximation approaches that yield tractable constraint expressions and ensure the convexity of the feasible set.

\section{Convex Approximations}

In this section, we present the convex approximation approach [28] in its general form and then, we extend our analysis with the study of two examples namely; Chernoff and Chebyshev-based approaches. Let $\phi: \mathbb{R} \rightarrow \mathbb{R}$ a nonnegative, nondecreasing, and convex function satisfying $\phi(z)>\phi(0)=1$ for all $z>0$. Then, for each $i \in\{1, \ldots, T\}$ and $n \in\{1, \ldots, N\}$, it follows that for all $\alpha>0$ :

$$
P\left(f_{i, n}\left(\mathbf{q}, \mathbf{Q}^{\mathbf{0}}\right)>0\right) \leq \mathbb{E}\left[\phi\left(\frac{f_{i, n}\left(\mathbf{q}, \mathbf{Q}^{\mathbf{0}}\right)}{\alpha}\right)\right]
$$

and

$$
P\left(g_{i, n}\left(\mathbf{q}, \mathbf{Q}^{\mathbf{0}}\right)>0\right) \leq \mathbb{E}\left[\phi\left(\frac{g_{i, n}\left(\mathbf{q}, \mathbf{Q}^{\mathbf{0}}\right)}{\alpha}\right)\right] .
$$

Hence, we deduce that if the right-hand sides of (20) and (21) are lower bounded by $1-\xi_{i, n}$ and $1-\nu_{i, n}$, respectively, then the constraints in (18) and (19) will be satisfied. Moreover, it was shown in [28] that the previous statement could be further strengthened by:

$$
\begin{aligned}
& \inf _{\alpha>0}\left(\alpha \mathbb{E}\left[\phi\left(\frac{f_{i, n}\left(\mathbf{q}, \mathbf{Q}^{\mathbf{0}}\right)}{\alpha}\right)\right]-\alpha\left(1-\xi_{i, n}\right)\right) \leq 0 \\
& \Longrightarrow P\left(f_{i, n}\left(\mathbf{q}, \mathbf{Q}^{\mathbf{0}}\right)>0\right) \leq 1-\xi_{i, n}
\end{aligned}
$$

and

$$
\begin{aligned}
& \inf _{\alpha>0}\left(\alpha \mathbb{E}\left[\phi\left(\frac{g_{i, n}\left(\mathbf{q}, \mathbf{Q}^{\mathbf{0}}\right)}{\alpha}\right)\right]-\alpha\left(1-\nu_{i, n}\right)\right) \leq 0 \\
& \Longrightarrow P\left(g_{i, n}\left(\mathbf{q}, \mathbf{Q}^{\mathbf{0}}\right)>0\right) \leq 1-\nu_{i, n}
\end{aligned}
$$

Thus, a conservative approximation of the optimization problem (17)-(19) is given as follows [28]: $\forall n \in\{1, \ldots, N\}$

$$
\begin{aligned}
& \underset{\tilde{q}_{i, n}^{0}, q_{i, n}^{0}, q_{i, n}^{1}}{\operatorname{maximize}} \sum_{i=1}^{T}\left(c_{i}^{0} \tilde{q}_{i, n}^{0}-c_{i}^{1} q_{i, n}^{1}\right) \\
& \text { subject to }(10),(13), \\
& \inf _{\alpha>0}\left(\alpha \mathbb{E}\left[\phi\left(\frac{f_{i, n}\left(\mathbf{q}, \mathbf{Q}^{\mathbf{0}}\right)}{\alpha}\right)\right]-\alpha\left(1-\xi_{i, n}\right)\right) \leq 0, \forall i, \\
& \inf _{\alpha>0}\left(\alpha \mathbb{E}\left[\phi\left(\frac{g_{i, n}\left(\mathbf{q}, \mathbf{Q}^{\mathbf{0}}\right)}{\alpha}\right)\right]-\alpha\left(1-\nu_{i, n}\right)\right) \leq 0, \forall i .
\end{aligned}
$$

The feasible set of this maximization problem is included in the one of (17)-(19). Thus, its optimal objective function is upper bounded by the optimal objective function of (17)(19). Moreover, given that the objective function is linear and the fact that all constraints in the optimization problem (9)(13) are affine functions for each realization of the generated energy of each internal retailer, it was shown in [28] that (24)(26) is a convex problem. Hence, the resolution of (24)-(26) can be easily performed using convex optimization algorithms such as the interior-point method. Furthermore, the constraints (25) and (26) are given by tractable expressions for particular choices of the function $\phi(\cdot)$. In the following two subsections, two choices of the function $\phi(\cdot)$ are presented.

\section{A. Chernoff Bound-Based Approach}

In this section, we apply the above conservative convex approximation using $\phi(x)=\exp (x), x \in \mathbb{R}$. This is known as the Chernoff bound. Assume that, for each BS $n \in\{1, \ldots, N\}$, the RVs $\left\{Q_{i, n}^{0}\right\}_{i=1}^{T}$ are independent and $M_{i, n}(\cdot)$ are their corresponding MGFs. The maximization problem (24)-(26) is 
given by: $\forall n \in\{1, \ldots, N\}$

$$
\underset{\tilde{q}_{i, n}^{0}, q_{i, n}^{0}, q_{i, n}^{1}}{\operatorname{maximize}} \sum_{i=1}^{T}\left(c_{i}^{0} \tilde{q}_{i, n}^{0}-c_{i}^{1} q_{i, n}^{1}\right)
$$

subject to $(10),(13)$,

$$
\begin{aligned}
& \inf _{\alpha>0}\left(\alpha \exp \left(\frac{\sum_{j=1}^{i}\left(q_{j, n}^{0}+\tilde{q}_{j, n}^{0}\right)}{\alpha}\right) \prod_{j=1}^{i} M_{j, n}\left(-\frac{1}{\alpha}\right)\right. \\
& \left.-\alpha\left(1-\xi_{i, n}\right)\right) \leq 0, \forall i, \\
& \inf _{\alpha>0}\left(\alpha \prod_{j=1}^{i} M_{j, n}\left(\frac{1}{\alpha}\right) \exp \left(-\frac{\sum_{j=1}^{i}\left(q_{j, n}^{0}+\tilde{q}_{j, n}^{0}\right)+\bar{C}_{n}}{\alpha}\right)\right. \\
& \left.-\alpha\left(1-\nu_{i, n}\right)\right) \leq 0, \forall i .
\end{aligned}
$$

The main feature of the above optimization problem is that it only depends on the individual MGFs of the RE generation $\mathrm{RVs}$ for each internal retailer and at each time period. More precisely, it does not need the knowledge of the distribution of the sum $\sum_{j=1}^{i} Q_{j, n}^{0}$ which is difficult to obtain in general.

As it was mentioned before, the above Chernoff approximation is an explicit convex optimization problem [28]. Moreover, the constraints (28) and (29) are efficiently computable using convex optimization algorithms [27].

\section{B. Chebyshev Bound-Based Approach}

In this section, we discuss the second approach based on the Chebyshev bound with $\phi(x)=[\max (x+1,0)]^{2}$. Note that with this choice of $\phi(\cdot)$, the evaluation of the expectations in (25) and (26) might be a difficult task and requires often the use of MC method. In order to avoid the high complexity incurred by the use of MC computation, we employ a more conservative choice of $\phi(\cdot)$ corresponding to $\phi(x)=(x+1)^{2}$. Note that this choice violates the nondecreasing property that $\phi(\cdot)$ should have in order to prove the convexity of the approximate optimization problem (24)-(26). However, given that the constraints in the original problem (9)-(13) are affine for each realization, we can easily prove that, in this particular case the optimization problem (24)-(26) is still convex provided the convexity and the non-negativity of $\phi(\cdot)$. Interestingly, for this particular choice of $\phi(\cdot)$, the constraints in (25) and (26) could be further simplified [28]. In fact, with $\phi(x)=(1+x)^{2}$, the left-hand side terms of the constraints in (25) and (26) have the following expressions:

$$
\begin{aligned}
& \alpha \mathbb{E}\left[\phi\left(\frac{f_{i, n}\left(\mathbf{q}, \mathbf{Q}^{\mathbf{0}}\right)}{\alpha}\right)\right]-\alpha\left(1-\xi_{i, n}\right) \\
& =\frac{\mathbb{E}\left[f_{i, n}^{2}\left(\mathbf{q}, \mathbf{Q}^{\mathbf{0}}\right)\right]}{\alpha}+2 \mathbb{E}\left[f_{i, n}\left(\mathbf{q}, \mathbf{Q}^{\mathbf{0}}\right)\right]+\xi_{i, n} \alpha
\end{aligned}
$$

and

$$
\begin{aligned}
& \alpha \mathbb{E}\left[\phi\left(\frac{g_{i, n}\left(\mathbf{q}, \mathbf{Q}^{\mathbf{0}}\right)}{\alpha}\right)\right]-\alpha\left(1-\nu_{i, n}\right) \\
& =\frac{\mathbb{E}\left[g_{i, n}^{2}\left(\mathbf{q}, \mathbf{Q}^{\mathbf{0}}\right)\right]}{\alpha}+2 \mathbb{E}\left[g_{i, n}\left(\mathbf{q}, \mathbf{Q}^{\mathbf{0}}\right)\right]+\nu_{i, n} \alpha
\end{aligned}
$$

After simple manipulations, the optimal values of $\alpha$ solving (30) and (31) are, respectively, expressed as follows:

$$
\alpha_{i, n}^{*}=\left(\frac{\mathbb{E}\left[\left(\sum_{j=1}^{i}\left(q_{j, n}^{0}+\tilde{q}_{j, n}^{0}\right)-\sum_{j=1}^{i} Q_{j, n}^{0}\right)^{2}\right]}{\xi_{i, n}}\right)^{\frac{1}{2}}
$$

and

$$
\alpha_{i, n}^{*}=\left(\frac{\mathbb{E}\left[\left(\sum_{j=1}^{i} Q_{j, n}^{0}-\sum_{j=1}^{i}\left(q_{j, n}^{0}+\tilde{q}_{j, n}^{0}\right)-\bar{C}_{n}\right)^{2}\right]}{\nu_{i, n}}\right)^{\frac{1}{2}}
$$

Thus, upon plugging these values in (25) and (26), the approximate optimization problem (24)-(26) is re-written as: $\forall n \in\{1, \ldots, N\}$

$$
\begin{aligned}
& \underset{\tilde{q}_{i, n}^{0}, q_{i, n}^{0}, q_{i, n}^{1}}{\operatorname{maximize}} \sum_{i=1}^{T}\left(c_{i}^{0} \tilde{q}_{i, n}^{0}-c_{i}^{1} q_{i, n}^{1}\right) \\
& \text { subject to }(10),(13), \\
& \mathbb{E}\left[\sum_{j=1}^{i}\left(q_{j, n}^{0}+\tilde{q}_{j, n}^{0}\right)-\sum_{j=1}^{i} Q_{j, n}^{0}\right] \\
& +\left(\xi_{i, n} \mathbb{E}\left[\left(\sum_{j=1}^{i}\left(q_{j, n}^{0}+\tilde{q}_{j, n}^{0}\right)-\sum_{j=1}^{i} Q_{j, n}^{0}\right)^{2}\right]\right)^{\frac{1}{2}} \leq 0, \forall i, \quad(35) \\
& \mathbb{E}\left[\sum_{j=1}^{i} Q_{j, n}^{0}-\left(\sum_{j=1}^{i}\left(q_{j, n}^{0}+\tilde{q}_{j, n}^{0}\right)\right)-\bar{C}_{n}\right] \\
& +\left(\nu_{i, n} \mathbb{E}\left[\left(\sum_{j=1}^{i} Q_{j, n}^{0}-\sum_{j=1}^{i}\left(q_{j, n}^{0}+\tilde{q}_{j, n}^{0}\right)-\bar{C}_{n}\right)^{2}\right]\right)^{\frac{1}{2}} \leq 0, \forall i
\end{aligned}
$$

Under the independence of $\left\{Q_{i, n}^{0}\right\}_{i=1}^{T}$ for each $n \in\{1, \ldots, N\}$, we clearly observe that the previous approximate maximization problem depends only on the first and second moments of the RVs $Q_{i, n}^{0}$. Let $\mu_{i, n}=\mathbb{E}\left[Q_{i, n}^{0}\right]$ and $\sigma_{i, n}^{2}=\operatorname{Var}\left[Q_{i, n}^{0}\right]$, then, by using the multinomial formula [29, Prop. 4.10], the maximization problem is expressed as follows: $\forall n \in$ 
$\{1, \ldots, N\}$

$\underset{\tilde{q}_{i, n}^{0}, q_{i, n}^{0}, q_{i, n}^{1}}{\operatorname{maximize}} \sum_{i=1}^{T}\left(c_{i}^{0} \tilde{q}_{i, n}^{0}-c_{i}^{1} q_{i, n}^{1}\right)$

subject to $(10),(13)$,

$\sum_{j=1}^{i}\left(q_{j, n}^{0}+\tilde{q}_{j, n}^{0}\right)-\sum_{j=1}^{i} \mu_{j, n}+$
$\left(\xi_{i, n}\left[\sum_{k_{1}+\ldots+k_{i}=2}\left(\begin{array}{c}2 \\ k_{1}, \ldots, k_{i}\end{array}\right) \prod_{1 \leq j \leq i} \mathbb{E}\left[X_{j, n}^{k_{j}}\right]\right]\right)^{\frac{1}{2}} \leq 0, \forall i$,

$$
\begin{aligned}
& \sum_{j=1}^{i} \mu_{j, n}^{0}-\sum_{j=1}^{i}\left(q_{j, n}^{0}+\tilde{q}_{j, n}^{0}\right)-\bar{C}_{n}+ \\
& \left(\nu_{i, n}\left[\sum_{k_{1}+\ldots+k_{i}=2}\left(\begin{array}{c}
2 \\
k_{1}, \ldots, k_{i}
\end{array}\right) \prod_{1 \leq j \leq i} \mathbb{E}\left[Y_{j, n}^{k_{j}}\right]\right]\right)^{\frac{1}{2}} \leq 0, \forall i,
\end{aligned}
$$

where $X_{j, n}=q_{j, n}^{0}+\tilde{q}_{j, n}^{0}-Q_{j, n}^{0}, Y_{j, n}=Q_{j, n}^{0}-q_{j, n}^{0}-\tilde{q}_{j, n}^{0}-$ $\bar{C}_{n} / i$, and

$$
\begin{aligned}
& \mathbb{E}\left[X_{j, n}^{k}\right]= \\
& \begin{cases}q_{j, n}^{0}+\tilde{q}_{j, n}^{0}-\mu_{j, n}^{0} & \mathrm{k}=1, \\
\left(q_{j, n}^{0}+\tilde{q}_{j, n}^{0}\right)^{2}-2 \mu_{j, n}\left(q_{j, n}^{0}+\tilde{q}_{j, n}^{0}\right)+\mu_{j, n}^{2}+\sigma_{j, n}^{2} & \mathrm{k}=2,\end{cases}
\end{aligned}
$$

and

$$
\begin{aligned}
& \mathbb{E}\left[Y_{j, n}^{k}\right]= \\
& \begin{cases}\mu_{j, n}^{0}-q_{j, n}^{0}-\tilde{q}_{j, n}^{0}-\frac{\bar{C}_{n}}{i} & \mathrm{k}=1, \\
\left(q_{j, n}^{0}+\tilde{q}_{j, n}^{0}+\frac{\bar{C}_{n}}{i}\right)^{2}-2 \mu_{j, n}\left(q_{j, n}^{0}+\tilde{q}_{j, n}^{0}+\frac{\bar{C}_{n}}{i}\right) & \mathrm{k}=2 . \\
+\mu_{j, n}^{2}+\sigma_{j, n}^{2} & \end{cases}
\end{aligned}
$$

The term $\left(\begin{array}{c}n \\ k_{1}, k_{2}, . ., k_{m}\end{array}\right)$ is the multinomial coefficient defined as:

$$
\left(\begin{array}{c}
n \\
k_{1}, k_{2}, . ., k_{m}
\end{array}\right)=\frac{n !}{k_{1} ! k_{2} ! \ldots k_{m} !} .
$$

Compared to the Chernoff-based approach, the Chebyshevbased approach exhibits less implementation complexity. This is because the constraints in the Chebyshev-based approach are given by explicit expressions, whereas the ones corresponding to the Chernoff-based approach are themselves the outputs of the minimization problems given in (28) and (29). Regarding their efficiency in making energy procurement decisions, the Chebyshev-based approach presents a lack of information about the uncertainty of the RE generation compared to the Chernoff-based approach. More precisely, it is only based on the knowledge of the first and second moments of the RE generation, whereas the latter one is based on the knowledge of the MGFs of the RE distributions. Thus, it is expected that the Chernoff-based approach will exhibit much higher performances than those of the one based on Chebyshev approximation in terms of energy procurement. The last statement will be confirmed in the following section.

\section{RESUlts AND Discussion}

In this section, we apply the two conservative convex approximations described in the previous section to optimize the energy procurement for one-day operation cycle. Given that the formulated optimization problem is separable in the sense that it can be solved for all BSs one by one, we restrict our analysis to the case of $N=1$. This will facilitate the interpretation of the developed results. Hence, the subscript $n$ can be dropped in all of the previous notations.

The simulation parameters are given as follows. A one-day operation cycle is divided into 6 time periods, each of 4 hours $(\Delta t=4)$. Note that this choice is only made to facilitate the analysis of the simulation results. The energy costs $c^{0}$ and $c^{1}$, and the consumed energy $E$ are considered as Heaviside step functions. The energy costs $c^{0}$ and $c^{1}$ are chosen such that $c_{i}^{0}$ is strictly less than $c_{i}^{1}$ for each time period. Moreover, the consumed energy $E$ follows, at each time period, the model in (1) with parameters $a=2.6, P_{u}=1 \mathrm{~W}$, and $b=56 \mathrm{~W}$. The RE generation $Q_{i}^{0}$ at a given time period $i$ is chosen to be uniformly distributed in the interval $\left[m_{i}, M_{i}\right]$ and independent from the generation of the other time periods. The average of $Q_{i}^{0}$ follows the wind energy model in [30] and is equal to $\frac{1}{2} \rho A V_{u}^{3} C_{p} \Delta t$ with air density $\rho=1.225 \mathrm{kgm}^{-3}$, blade swept area $A=\pi \mathrm{m}^{2}$, wind speed $V_{u}^{3}$ which varies at each time interval, and conversion coefficient $C_{p}=0.12$. For a given confidence measure $\eta$, the parameters $\xi_{i}$ and $\nu_{i}$ satisfy $\xi_{i}=\nu_{i}=1-\frac{1-\eta}{2 T}$, for all $i \in\{1, \ldots, T\}$. This choice is mainly motivated by the fact that the mobile operator accords the same confidence level to all constraints. Such a choice would be the most fair choice in the sense that it enables mobile operators to deal with all of the constraints in a similar manner by according to them the same risk level. The simulation parameters are summarized in Table I

TABLE I: Simulation parameters

\begin{tabular}{|l||l|l|l|l|l|l|}
\hline & 1 & 2 & 3 & 4 & 5 & 6 \\
\hline \hline$E_{i}(\mathrm{Wh})$ & 360 & 380 & 520 & 650 & 570 & 460 \\
\hline$c_{i}^{0}$ & 1 & 1 & 1.3 & 1.3 & 1.3 & 1 \\
\hline$c_{i}^{1}$ & 1.25 & 1.25 & 1.5 & 2 & 1.75 & 1.25 \\
\hline$m_{i}(\mathrm{Wh})$ & 300 & 300 & 700 & 600 & 400 & 400 \\
\hline$M_{i}(\mathrm{Wh})$ & 400 & 400 & 800 & 700 & 500 & 500 \\
\hline
\end{tabular}

As a first experiment, we plot in Fig. 2 the amounts of energy procured from the $\operatorname{RE~} q_{i}^{0}$, sold to the SG $\tilde{q}_{i}^{0}$, and procured from the SG $q_{i}^{1}$ using the Chernoff and the Chebyshev convex approximation approaches in the presence of uncertainty. In the same figure, these quantities are also plotted in the deterministic setting, i.e., the $\mathrm{RE}$ generation $Q_{i}^{0}$ is deterministic and equals to $\frac{m_{i}+M_{i}}{2}$ for each time period. The confidence level in this experiment is $\eta=0.9$, whereas the capacity of storage $\bar{C}$ is chosen to be equal to $2000 \mathrm{Wh}$. The storage constraint is relaxed in this simulation to more focus on the procurement management over the cycles, the impact of $\bar{C}$ is studied in Fig. 4.

From this figure, we distinguish three different regimes. In the first two time periods, the three approaches have the same procurement strategy by procuring all the required energy from the SG. This observation is expected for two reasons. First, 


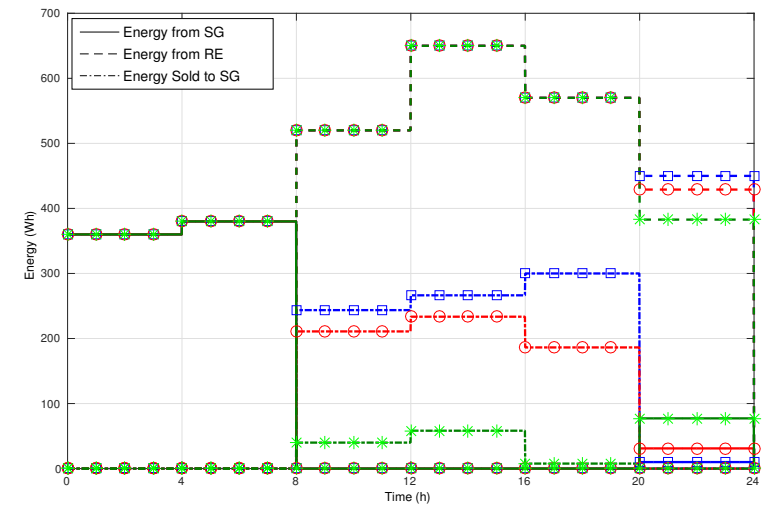

Fig. 2: Energy Procurement with $\eta=0.9, \bar{C}=2000 \mathrm{Wh}$, and simulation parameters in Table I. Deterministic approach in blue with square marker, Chernoff-based approach in red with circle marker, and Chebyshev-based approach in green with asterisk marker.

given that the price set by the SG in the next three time periods is higher than that of the first two periods (see Table I), the three approaches try to save the energy generated from the RE source so they can consume it in the next three time periods. Second, the price with which the mobile operator sells the energy to the SG will increase as well in these three periods. This also explains why the three approaches are saving energy so that they can sell a part of it to the SG in the next three time periods. The two previous statements are clearly confirmed in the next regime (from $8 \mathrm{~h}$ to $20 \mathrm{~h}$ ). In fact, the generated RE in these three periods and the stored quantities of the previous two time periods are used to fulfill the energy requirement. Moreover, the three approaches sell the remaining RE in these three time periods since the corresponding prices are attractive. Finally, in the remaining time period, the three approaches behave again similarly. In fact, as the quantity of RE was used in the previous time periods, these approaches will fulfill the energy requirement by consuming all the RE generated at this time period and buy the remaining quantity from the SG.

Fig. 2 also reveals that the energy procurement strategies of both Chernoff and Chebyshev approaches are more conservative compared to that of the deterministic case. In other words, with the risk level that corresponds to $10 \%$ in this experiment, the Chernoff and the Chebyshev-based approaches become more risk-aware than the deterministic approach in the sense that they are consuming less amount of RE. However, it should be noted that the high performance of the deterministic approach has to be balanced with its high risk, which must be bigger than $10 \%$, of being over estimating the real generation of the RE. For the sake of illustration, the energy generated from the RE source in the whole period is $\sum_{i=1}^{6} \frac{m_{i}+M_{i}}{2}=3000 \mathrm{Wh}$ in the deterministic case, whereas only $2800 \mathrm{Wh}$ and $2285 \mathrm{Wh}$ have been employed by Chernoff and Chebyshev approaches, respectively, in order to meet the risk requirement.

Furthermore, Fig. 2 shows that out of the two conservative

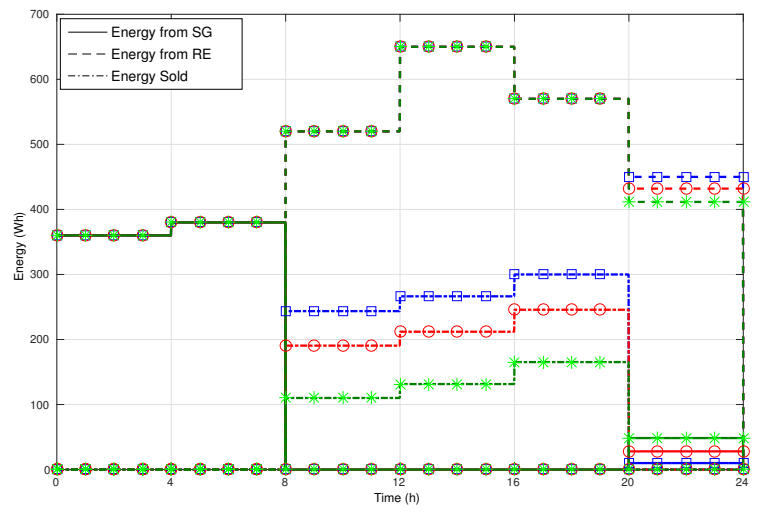

Fig. 3: Energy Procurement with $\eta=0.7, \bar{C}=2000 \mathrm{Wh}$, and simulation parameters in Table I. Deterministic approach in blue with square marker, Chernoff-based approach in red with circle marker, and Chebyshev-based approach in green with asterisk marker.

convex approximations, it is the Chernoff approach that has a more efficient energy procurement strategy. More precisely, while both approaches satisfy the $10 \%$ risk level requirement, the Chernoff-based approach procures a bigger amount from the RE source than the Chebyshev one. For instance, it is clear from Fig. 2 that, in the period between $8 \mathrm{~h}$ and $20 \mathrm{~h}$, the latter approach sells less amount of energy than that of the former. Moreover, in the last period, the amount of energy bought from the SG in addition to the one procured from the RE source using the Chernoff-based approach are, respectively, less and higher than the ones obtained with the Chebyshevbased approach. Finally, another interesting result exhibited in Fig. 2 is that the amount of RE used by the Chebyshev approach in the whole period i.e., $2285 \mathrm{Wh}$, is less than the minimum amount of RE which is $\sum_{i=1}^{6} m_{i}=2700 \mathrm{Wh}$. The reason of this under estimation is related to the little amount of information that the Chebyshev-based approach knows about the RE distribution. Such a failure is avoided by the Chernoff approach because of its perfect knowledge about the RE distribution. The last statement goes in favor towards the efficiency of the Chernoff-based approach compared to the Chebyshev one. As a second experiment, we aim to study the effect of the risk level on the energy procurement of Chernoff- and Chebyshev-based approaches. To this end, we plot in Fig. 3 the same results shown in Fig. 2 but with $\eta=0.7$. The simulation parameters are the same as in the previous experiment. In this setting, we clearly observe that both Chernoff- and Chebyshev-based approaches are procuring the energy more efficiently than in the previous experiment. Such an observation is expected since the bigger the risk level is, the higher the profit is. More precisely, by increasing the risk level, the amounts of RE, that the two approaches are expecting to be generated, will certainly increase and hence, their energy procurement strategy will be more efficient. For instance, in the period between $8 \mathrm{~h}$ and $20 \mathrm{~h}$, the Chernoff and the Chebyshev methods sell more quantities of RE than 
in the previous experiment. Moreover, to fulfill the energy requirement, the two approaches use more quantity from the RE source in the last time period. Note also that the Chernoff-based approach outperforms again the Chebyshevbased approach. From a mobile operator point of view, the confidence parameter $\eta$ is very important in the procurement decision. Indeed, in the case of error in the estimation of the $\mathrm{RE}$ generation at a given time period, the corresponding BS will not be completely powered and hence, some users will not be served due to the lack of power which leads to a high outage rate. In this case, the mobile operator has to increase the confidence level in order to meet its BS's power requirement and guarantee the safe network operation by buying extra energy from the electrical grid. In the considered scenarios given in Fig. 2 and Fig. 3 and following the Chernoff-based approach, increasing the confidence level from 0.7 to 0.9 , will cause approximately a loss of $22.74 \%$, as the profit goes from -117.26 to -143.92 .

We aim now to analyze the implementation complexity of the two proposed convex approximations. To this end, we provide in Table II the computational time given by the Chernoff- and the Chebyshev-based approaches for two values of the confidence level $\eta$. As expected, the Chebyshev-based approach exhibits less complexity than the Chernoff-based method. As it was mentioned before, this is due to the fact that the constraints of the Chebyshev optimization program are explicitly given whereas the constraints in the Chernoffbased optimization problem are themselves the outputs of other optimization problems.

TABLE II: Complexity Analysis

\begin{tabular}{|c|c|c||c|c|}
\hline & \multicolumn{2}{|c||}{ Chebyshev } & \multicolumn{2}{c|}{ Chernoff } \\
\hline$\eta$ & cpu time & Profit & cpu time & Profit \\
\hline 0.9 & 5.61 & -883.91 & 382.89 & -143.91 \\
0.7 & 5.13 & -456.64 & 335.48 & -117.26 \\
\hline
\end{tabular}

However, as explained previously, the previous statement has to be balanced with the efficiency of the Chernoff-based approach compared to the Chebyshev method in terms of energy procurement decision.

In the last experiment, we investigate the effect of varying the amount of consumed energy and the capacity of storage $\bar{C}$ on the energy procurement. More precisely, we vary the amount of the consumed energy $E$ in Table I via multiplying it by a factor $\beta$. Then, we plot in Fig. 4 the profit given by the deterministic and the Chernoff approaches as a function of $\beta$ for three different values of $\bar{C}$. We observe, from this figure, that the profit is a decreasing function of $\beta$. This is because the smaller the energy consumption is, the less (and/or the higher) the amounts of energy bought from (and/or sold to) the SG are. Moreover, we distinguish two regimes regarding the impact of varying the storage capacity on the profit. For small values of $\beta$, i.e., between 0.8 and 1.2 , we point out that the profit is approximately the same for the three values of $\bar{C}$. Then, as $\beta$ increases, the impact of $\bar{C}$ becomes clearly obvious. In fact, as the consumed energy increases, the optimization program tries to save as much as RE so it can use it in the period between $8 \mathrm{~h}$ to $20 \mathrm{~h}$ where the prices set by the SG are

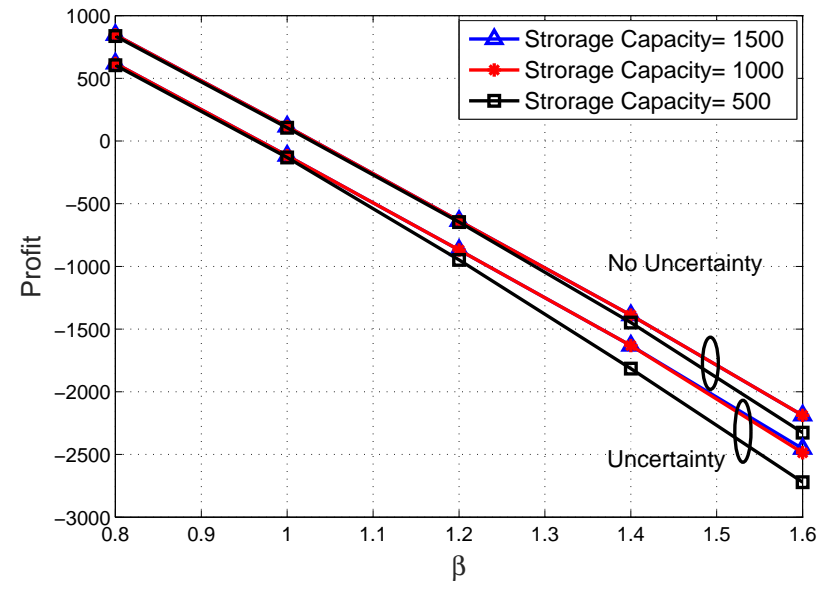

Fig. 4: Daily profit versus the amount of energy consumed by the cellular networks for different values of the storage capacity. The confidence level is $\eta=0.7$ and the simulation parameters are in Table I.

high. Hence, increasing the storage capacity when the energy consumption is high will certainly enhance the profit. Note also that the Chernoff-based approach is more sensitive to $\bar{C}$ than the deterministic setting.

\section{CONCLUSIONS}

In this paper, we developed an energy procurement framework for cellular networks for uncertain renewable energy generation. We formulated a profit optimization problem based on chance constrained optimization to handle the renewable energy randomness. We employed a conservative convex approximation approach to the chance constrained problem to make its resolution more tractable. We proposed two convex approximation methods based on the Chebyshev and the Chernoff bounds. In the simulation results, we investigated the energy procurement process given by first the deterministic setting (i.e., without uncertainty) and second by the Chebyshev- and the Chernoff-based approaches (i.e., in the presence of uncertainty). It was shown that with the Chernoffbased approach, the cellular networks procure energy more efficiently than with the Chebyshev-based approach. Moreover, it was shown that the risk level and the amount of consumed energy have significant impacts on the mobile operator's procurement decision during the whole cycle. A possible future work is to enable a dependence among the base stations in the procurement decision. For instance, it may be interesting to allow the base stations to exchange with each other the surplus of renewable energy generated locally. It is also interesting to complement the current work by integrating optimized resource allocation scheme aiming at minimizing the network energy consumption while maintaining the required quality of service.

\section{REFERENCES}

[1] O. Arouk, A. Ksentini, and T. Taleb, "Group paging-based energy saving for massive MTC accesses in LTE and beyond networks," IEEE Journal 
on Selected Areas in Communications, vol. 34, no. 5, pp. 1086-1102, May 2016.

[2] C. A. Chan, W. Li, S. Bian, I. Chih-Lin, A. F. Gygax, C. Leckie, M. Yan, and K. Hinton, "Assessing network energy consumption of mobile applications," IEEE Communications Magazine, vol. 53, no. 11, pp. 182-191, Nov. 2015.

[3] S. K. Korotky, "Semi-empirical description and projections of Internet traffic trends using a hyperbolic compound annual growth rate," Bell Labs Technical Journal, vol. 18, no. 3, pp. 5-21, Dec. 2013.

[4] A. Fehske, G. Fettweis, J. Malmodin, and G. Biczok, "The global footprint of mobile communications: The ecological and economic perspective," IEEE Communications Magazine, vol. 49, no. 8, pp. 55-62, Aug. 2011.

[5] M. Z. Shakir, K. A. Qaraqe, H. Tabassum, M. S. Alouini, E. Serpedin, and M. A. Imran, "Green heterogeneous small-cell networks: toward reducing the $\mathrm{CO}_{2}$ emissions of mobile communications industry using uplink power adaptation," IEEE Communications Magazine, vol. 51, no. 6, pp. 52-61, Jun. 2013.

[6] H. Ghazzai, E. Yaacoub, A. Kadri, H. Yanikomeroglu, and M. S. Alouini, "Next-generation environment-aware cellular networks: Modern green techniques and implementation challenges," IEEE Access, vol. 4, pp. 5010-5029, Sept. 2016.

[7] Y. Yan, Y. Qian, H. Sharif, and D. Tipper, "A survey on smart grid communication infrastructures: Motivations, requirements and challenges," IEEE Communications Surveys Tutorials, vol. 15, no. 1, pp. 5-20, First Quarter 2013.

[8] F. Li, W. Qiao, H. Sun, H. Wan, J. Wang, Y. Xia, Z. Xu, and P. Zhang, "Smart transmission grid: Vision and framework," IEEE Transactions on Smart Grid, vol. 1, no. 2, pp. 168-177, Sept. 2010.

[9] F. Davoli, M. Repetto, C. Tornelli, G. Proserpio, and F. Cucchietti, "Boosting energy efficiency through smart grids," Tech. rep., ITU's Telecommunication Standardization, Sept. 2012.

[10] Nokia Solutions and Networks, "Technology vision 2020 flatten network energy consumption," White Paper, Dec. 2013.

[11] T. Han and N. Ansari, "Powering mobile networks with green energy," IEEE Wireless Communications, vol. 21, no. 1, pp. 90-96, Feb. 2014.

[12] V. Chamola and B. Sikdar, "Solar powered cellular base stations: current scenario, issues and proposed solutions," IEEE Communications Magazine, vol. 54, no. 5, pp. 108-114, May. 2016.

[13] H. Liang, A. K. Tamang, W. Zhuang, and X. S. Shen, "Stochastic information management in smart grid," IEEE Communications Surveys Tutorials, vol. 16, no. 3, pp. 1746-1770, Third Quarter 2014.

[14] D. Li, W. Saad, I. Guvenc, A. Mehbodniya, and F. Adachi, "Decentralized energy allocation for wireless networks with renewable energy powered base stations," IEEE Transactions on Communications, vol. 63, no. 6, pp. 2126-2142, June 2015.

[15] T. Han and N. Ansari, "Provisioning green energy for base stations in heterogeneous networks," IEEE Transactions on Vehicular Technology, vol. 65, no. 7, pp. 5439-5448, July 2016.

[16] H. Al Haj Hassan, A. Pelov and L. Nuaymi, "Integrating cellular networks, smart grid, and renewable energy: Analysis, architecture, and challenges," IEEE Access, vol. 3, pp. 2755-2770, Dec. 2015.

[17] H. Ghazzai and A. Kadri, "Joint demand-side management in smart grid for green collaborative mobile operators under dynamic pricing and fairness setup," IEEE Transactions on Green Communications and Networking, vol. 1, no. 1, pp. 74-88, Mar. 2017.

[18] T. Han and N. Ansari, "On optimizing green energy utilization for cellular networks with hybrid energy supplies," IEEE Transactions on Wireless Communications, vol. 12, no. 8, pp. 3872-3882, Aug. 2013.

[19] M. J. Farooq, H. Ghazzai, and A. Kadri, "Optimized energy procurement for cellular networks powered by smart grid based on stochastic geometry," in IEEE Globecom Workshops (GC Wkshps), San Diego, CA, USA, Dec. 2015.

[20] K. Ponnambalam, Y. E. Saad, M. Mahootchi, and A. W. Heemink, "Comparison of methods for battery capacity design in renewable energy systems for constant demand and uncertain supply," in the 7th International Conference on the European Energy Market (EEM'10), Madrid, Spain, Jun. 2010.

[21] I. Ahmed, A. Ikhlef, D. W. K. Ng, and R. Schober, "Power allocation for an energy harvesting transmitter with hybrid energy sources," IEEE Transactions on Wireless Communications, vol. 12, no. 12, pp. 62556267, Dec. 2013.

[22] D. W. K. Ng, E. S. Lo, and R. Schober, "Energy-efficient resource allocation in ofdma systems with hybrid energy harvesting base station," IEEE Transactions on Wireless Communications, vol. 12, no. 7, pp. 3412-3427, Jul. 2013.
[23] D. Niyato, X. Lu, and P. Wang, "Adaptive power management for wireless base stations in a smart grid environment," IEEE Wireless Communications, vol. 19, no. 6, pp. 44-51, Dec. 2012.

[24] M. J. Farooq, H. Ghazzai, A. Kadri, H. ElSawy, and M. S. Alouini, "A hybrid energy sharing framework for green cellular networks," IEEE Transactions on Communications, vol. 65, no. 2, pp. 918-934, Feb. 2017.

[25] N. B. Rached, H. Ghazzai, A. Kadri, and M. S. Alouini, "Optimized energy procurement for cellular networks with uncertain renewable energy generation," in Proc. of IEEE Global Communications Conference (GLOBECOM), Washington, DC, USA, Dec. 2016.

[26] G. Auer and et al, "D2.3 v2: Energy efficiency analysis of the reference systems, areas of improvements and target breakdown," INFSO-ICT247733 EARTH (Energy Aware Radio and NeTwork TecHnologies), Tech. Rep., Jan. 2012.

[27] S. Boyd and L. Vandenberghe, Convex Optimization. Cambridge University Press, 2004.

[28] A. Nemirovski and A. Shapiro, "Convex approximations of chance constrained programs," SIAM J. on Optimization, vol. 17, no. 4, pp. 969-996, Dec. 2006.

[29] J. Gallier, "Some counting problems; multinomial coefficients, the principle of inclusion-exclusion, Sylvester's formula, the Sieve formula,' in Discrete Mathematics, ser. Universitext. Springer New York, 2011, pp. 205-255.

[30] A. W. Manyonge, R. M. Ochieng, F. N. Onyango, and J. M. Shichikha, "Mathematical modeling of wind turbine in a wind energy conversion system: Power coefficient analysis," Applied Mathematical Sciences, vol. 6, no. 91, pp. 4527-4536, Jan. 2012.

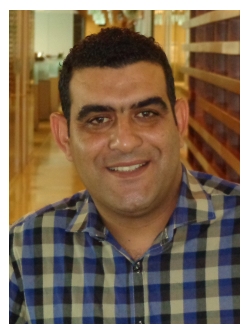

Nadhir Ben Rached was born in Nabeul, Tunisia. He received the Diplôme d'Ingénieur degree from the École Polytechnique de Tunisie, La Marsa, Tunisia, in 2012 and the M.S. degree in Applied Mathematics and Computational Science from King Abdullah University of Science and Technology, Thuwal, Saudi Arabia, in 2013, where he is currently working toward the Ph.D degree in Statistics. His current research interests include rare event simulation algorithms for the accurate performance analysis of wireless communication systems.

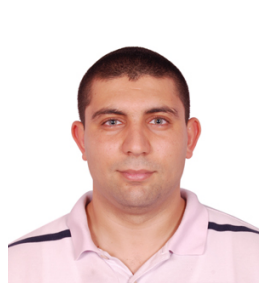

Hakim Ghazzai (S'12, M'15) was born in Tunisia. $\mathrm{He}$ is currently working as a research scientist at Qatar Mobility Innovations Center (QMIC), Doha, Qatar. He received his Ph.D degree in Electrical Engineering from King Abdullah University of Science and Technology (KAUST), Saudi Arabia in 2015. He received his Diplome d'Ingenieur in telecommunication engineering and Master of Science degree from the Ecole Superieure des Communications de Tunis (SUP'COM), Tunisia in 2010 and 2011, respectively. He is a recipient of appreciation for an exemplary reviewer for IEEE Wireless Communications Letters (WCL) in 2016. His general research interests include mobile and wireless networks, green communications, internet of things, and UAV-based communications. 


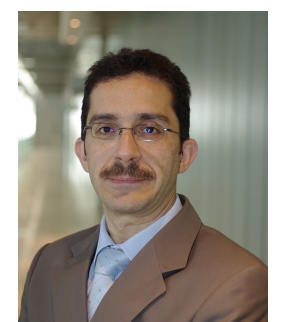

Abdullah Kadri (SM'16) received the M.E.Sc. and $\mathrm{Ph} . \mathrm{D}$. degrees in electrical engineering from the University of Western Ontario (UWO), London, ON, Canada, in 2005 and 2009, respectively. Between 2009 and 2012, he worked as a Research Scientist at Qatar Mobility Innovations Center (QMIC), Qatar University. In 2013, he became a Senior R\& D Expert and the Technology Lead at QMIC focusing on R\& D activities related to intelligent sensing and monitoring using mobility sensing. His research interests include wireless communications, wireless sensor networks for harsh environment applications, indoor localization, internet-of-things, green communication, applications of UAVs, and smart sensing. He is the recipient of the Best Paper Award at the WCNC Conference in 2014 .

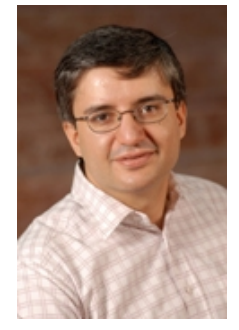

Mohamed-Slim Alouini (S'94, M'98, SM'03, F'09) was born in Tunis, Tunisia. He received the Ph.D. degree in Electrical Engineering from the California Institute of Technology (Caltech), Pasadena, CA, USA, in 1998. He served as a faculty member in the University of Minnesota, Minneapolis, MN, USA, then in the Texas A\&M University at Qatar, Education City, Doha, Qatar before joining King Abdullah University of Science and Technology (KAUST), Thuwal, Makkah Province, Saudi Arabia as a Professor of Electrical Engineering in 2009. His current research interests include the modeling, design, and performance analysis of wireless communication systems. 